\title{
Combined Channel Estimation and Turbo Equalization on Wireless Channels
}

\author{
Fu-Sheng Shiao, Desmond P. Taylor, Philippa A. Martin \\ Electrical and Computer Engineering Department, \\ University of Canterbury, Private Bag 4800, Christchurch, NEW ZEALAND. \\ Email: \{taylor, p.martin\}@elec.canterbury.ac.nz.
}

\begin{abstract}
To date most frequency-domain (FD) turbo equalization schemes assume ideal channel state information (CSI) is available. In this paper, a system combining FD turbo linear equalization with time-domain channel estimation is developed and evaluated for single-carrier modulation formats. The effect of estimated CSI on the equalizer form is shown. Performance results employing convolutionally encoded QPSK and 16-QAM transmissions show the efficacy of the proposed system and its capability to operate in different wireless scenarios.
\end{abstract}

\section{INTRODUCTION}

Single-carrier (SC) linear modulation techniques combined with frequency-domain (FD) equalization provide an alternative to multicarrier techniques for combating multipath fading in channels with large delay spread [1]. They tolerate frequency offset and have well controlled peak to average power ratio. They have comparable complexity to orthogonal frequency division multiplexing systems, and are more robust to synchronization errors [1].

If error correction coding is used, then information can be iteratively passed between the equalizer and decoder to improve performance. This is called turbo equalization [2]. Recently, several such schemes were proposed [3]-[10]. A time-domain (TD) scheme was described in [3] and a FD scheme was proposed in [3], [10]. They all require exact knowledge of the channel state information (CSI). Here, we employ the scheme of [10] as it is derived using fewer approximations than that of [3], is less complex and is usable with a variety of channel estimation schemes. Moreover, it is effective in highly dispersive channels.

There has been some research into turbo equalizers that do not require a priori knowledge of the CSI [11]-[13]. One approach is to iteratively minimize a cost function [11], [12]. This approach was employed in [9] to jointly estimate the channel in parallel with TD turbo equalization. Its disadvantage is that the variance of the estimation error is not easily obtained from the soft outputs of the soft-input soft-output (SISO) decoder. A similar TD scheme was given in [13]. It structured channel estimation as a Kalman state estimator by processing the soft information from the decoding process as part of the statistical description of the channel. However, it leads to a very complex structure, especially for high-level modulation formats.

Here, we investigate FD turbo equalization with estimated CSI at the receiver. We combine the FD turbo linear equalization (FD-TLE) algorithm of [10] with the low-complexity
TD channel estimation algorithm of [14]. This estimation scheme was used primarily because of its simplicity and relatively good performance in very slow fading. However, it is recognized that more accurate approaches, such as that of [15], would yield better performance, albeit with increased complexity. The novelty of the resulting scheme lies in the combination of adaptive TD channel estimation with FD turbo equalization. Furthermore, the scheme is extended to high level modulation formats and remains computationally feasible. Our derivation of FD-TLE for estimated CSI shows that imperfect estimates lead to bias terms in the equalizer matrix.

\section{SySTEM OVERVIEW}

A block diagram of the proposed system in complex baseband is shown in Fig. 1. Prior to transmission, a training sequence is used to train the channel estimator. The system is then switched to transmission mode where a further training sequence is embedded in the unique word (UW) prefix and suffix of each transmitted block. These are used by the channel estimator to track the CSI.

Data is encoded using a convolutional code ${ }^{1}$. Each group of $\log _{2} M$ consecutive encoded bits is Gray-mapped to an $M$ ary constellation point/ channel symbol $\left\{\mathcal{X}_{l} \mid l=0 \ldots M-1\right\}$. The resulting symbol stream at rate $f=1 / T$ is divided into non-overlapping blocks of length $N_{C S}$. Each block is interleaved to produce the block of channel symbols $\mathbf{x}_{N_{C S}}=\left[x_{0}, x_{1}, \ldots, x_{N_{C S}-1}\right]^{T}$. A UW of length $N_{U W}$ is appended to $\mathbf{x}_{N_{C S}}$ as a prefix and suffix to produce the sequence $\mathbf{x}_{N_{T}}=\left[x_{-N_{U W}}, \ldots, x_{-1}, x_{0}, x_{1}, \ldots, x_{N_{C S}-1}, x_{N_{C S}}\right.$, $\left.\ldots, x_{N_{C S}+N_{U W}-1}\right]^{T}$. This is passed through a root raised cosine filter with impulse response $p(t)$ and rolloff $\alpha$, truncated to span $L_{p}$ symbols. It is then transmitted over a frequency selective fading channel with impulse response $c(t)$, modeled as a delay line with $L_{c}$ independent symbol-spaced taps. We assume that $c(t)$ is slowly time varying, and changes negligibly over each transmitted block.

The received signal can be expressed as

$$
r(t)=\sum_{n=-N_{U W}}^{N_{U W}+N_{C S}-1} x_{n} h(t-n T)+w(t)
$$

where $h(t)$ is the composite channel impulse response spanning $L_{h}=L_{p}+L_{c}$ symbol periods and $w(t)$ is additive

\footnotetext{
${ }^{1} \mathrm{~A}$ variety of codes such as turbo or low density parity check (LDPC) codes could also be used.
} 


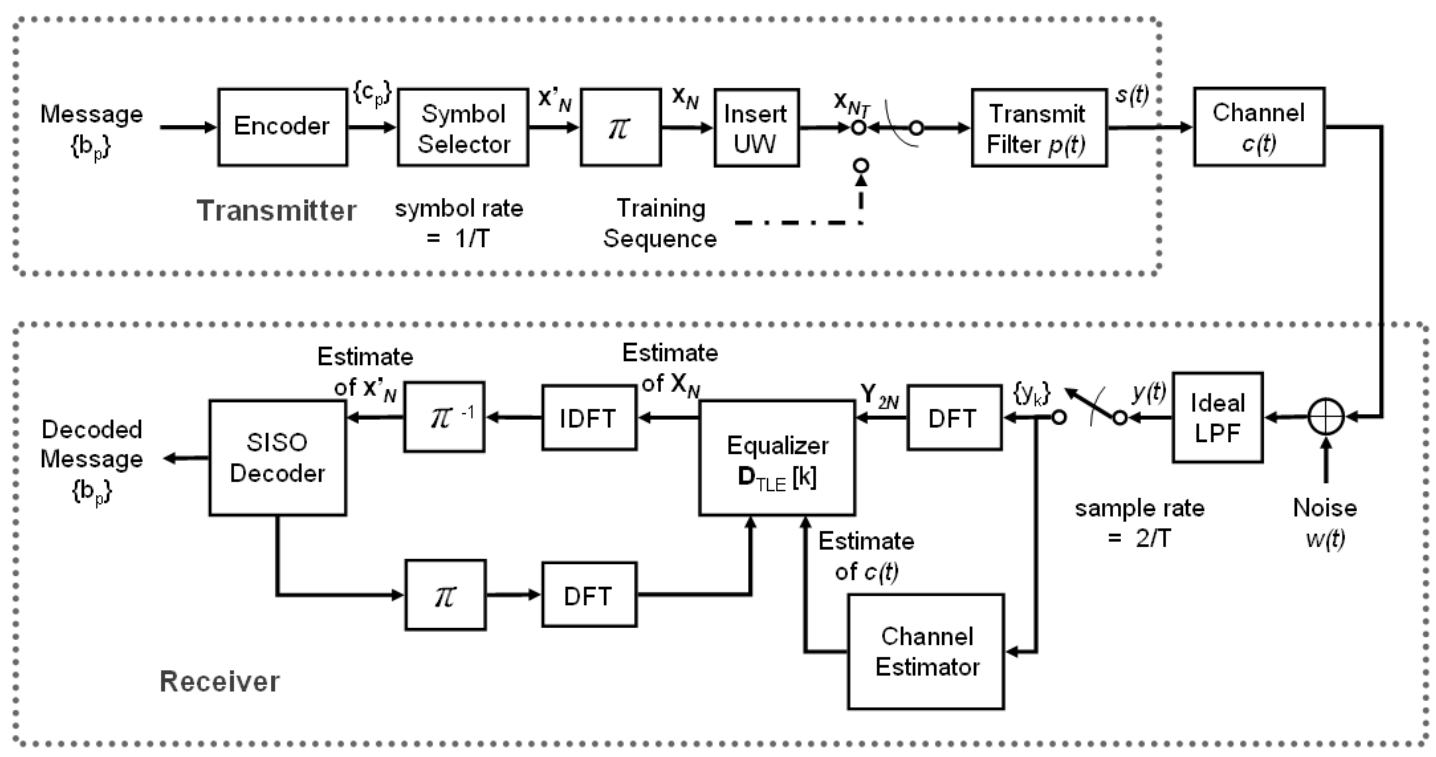

Fig. 1. FD-TLE with channel estimation.

white Gaussian noise (AWGN) with two-sided power spectral density $2 N_{0}$. The signal $r(t)$ is passed through an ideal lowpass filter (LPF) having two-sided bandwidth $1 / T$ and gain $\sqrt{T / 2}$ to produce the bandlimited random process

$$
y(t)=\sum_{n=-N_{U W}}^{N_{U W}+N_{C S}-1} \sqrt{\frac{T}{2}} x_{n} h(t-n T)+n(t),
$$

where $n(t)$ is Gaussian noise having zero-mean and two-sided power spectral density $N_{0} T$ for $|f| \leq 1 / T$. Following [10], an ideal LPF is used instead of a matched filter since the channel is random and unknown.

The signal $y(t)$ is sampled at rate $^{2} f_{s}=N_{s} / T$ and the samples corresponding to the UW prefix are used to update the CSI estimate before being discarded. The samples corresponding to the UW suffix are not removed as they are used to estimate the variance of the equalization error at the equalizer output [16]. The remaining samples of $y(t)$ correspond to the symbol block $\mathbf{x}_{N}=\left[x_{0}, x_{1}, \ldots, x_{N-1}\right]^{T}$, where $N=N_{U W}+N_{C S}$. These samples may be expressed as a $2 N$-dimensional complex vector, $\mathbf{y}_{2 N}=\left[y_{0}, y_{1}, \ldots, y_{2 N-1}\right]$, where for $i=0, \ldots, 2 N-1$

$$
y_{i}=y\left(t_{i}\right)=\frac{1}{\sqrt{2 N}} \sum_{k=-N}^{N} P_{k} X_{k} C_{k} \exp \left(j \frac{2 \pi k i}{2 N}\right)+n_{i},
$$

$t_{i}=i T / 2$ for $i=0, \ldots, 2 N-1, P_{k}=P(k / N T) / \sqrt{T}, X_{k}$ is the $k^{\text {th }}$ component of the vector $\mathbf{X}_{N}=\left[X_{0}, X_{1}, \ldots, X_{N-1}\right]^{T}$ resulting from the DFT of $\mathbf{x}_{N}, X_{-k}=X_{k}^{*}$ and $C_{k}=$ $C(k / N T)$. Note that $P(f)$ and $C(f)$ are the Fourier transforms of $p(t)$ and $c(t)$, respectively. The noise samples $n_{i}=$

\footnotetext{
${ }^{2}$ Although $N_{s}$ can take any integer value to accommodate Doppler, in most practical instances, $N_{s}=2$ is sufficient and is used here. Also the simulation results presented in Section IV use $N_{s}=2$.
}

$n\left(t_{i}\right)$ are independent identically distributed random variables having zero mean and variance $\sigma_{n}^{2}=2 N_{0}$.

The DFT block in Fig. 1 operates on $\mathbf{y}_{2 N}$, to produce the FD vector $\mathbf{Y}_{2 N}=\left[Y_{0}, Y_{1}, \ldots, Y_{2 N-1}\right]^{T}$, where [10]

$$
\begin{aligned}
Y_{k} & =R_{k} X_{k}+V_{k}, \\
R_{k} & = \begin{cases}P_{k} C_{k}, & k=0,1, \ldots, N \\
P_{k-2 N} C_{k-2 N}, & k=N+1, N+2, \ldots, 2 N-1 .\end{cases}
\end{aligned}
$$

The set of random variables $\left\{V_{k}\right\}$ is statistically equivalent to $\left\{n_{i}\right\}$. We may write (4) in matrix form as $\mathbf{Y}_{2 N}=\mathbf{R X}_{2 N}+$ $\mathbf{V}$, where $\mathbf{R}=\operatorname{diag}\left(R_{k}\right), \mathbf{X}_{2 N}=\left[\mathbf{X}_{N}^{T} \mid \mathbf{X}_{N}^{T}\right]^{T}$ and $\mathbf{V}=$ $\left[V_{0}, V_{1}, \ldots, V_{2 N-1}\right]^{T}$.

The equalizer filters $\mathbf{Y}_{2 N}$ by an $N \times 2 N$ matrix $\left.\mathbf{D}_{T L E}[\psi]\right|_{\psi=1}$ to produce the $N$-dimensional vector $\widehat{\mathbf{x}}_{N}$. This is passed through the IDFT block and a deinterleaver to produce the deinterleaved symbol estimate $\widehat{\mathbf{x}}_{N}^{\prime}$. Simulations were run to determine the distribution of the equalization error in $\widehat{\mathbf{x}}_{N}^{\prime}$. It was found to be close to Gaussian [16].

The estimate $\widehat{\mathbf{x}}_{N}^{\prime}$ is fed into the SISO decoder which generates both symbol a posteriori probabilities (APPs) and data estimates. The APPs are passed back to the equalizer through an interleaver and a DFT and used to adjust the equalizer matrix to $\left.\mathbf{D}_{T L E}[\psi]\right|_{\psi=2}$. An improved data estimate of $\mathbf{X}_{N}$ is then obtained and used for the next iteration. This process continues for some set number of iterations, following which the decoded data is obtained from the SISO decoder.

\section{FD-TLE With ChANNEl Estimation}

\section{A. Channel Estimation Using GLMS Channel Estimator}

We use the generalized least mean squares (GLMS) estimator of [14] as the channel estimator in Fig. 1. It is a simplification of the generalized recursive least squares 
(GRLS) estimator [15]. The GLMS was chosen because it may be more simply implemented than schemes such as the GRLS approach of [15] or a Kalman estimator [17]. In addition, in many situations, its performance is only slightly worse. We summarize it in the following.

To initiate channel estimation, a training sequence $\mathbf{d}_{\text {Train }}=$ $\left[d_{0-L_{h} / 2}, \ldots, d_{0}, \ldots, d_{N_{\text {Train }}+L_{h} / 2-1}\right]$ is transmitted, where $N_{\text {Train }}$ is the training sequence length and is chosen to ensure estimator convergence $\left(N_{\text {Train }}=156\right.$ was sufficient). The $L_{h}$ extra symbols in $\mathbf{d}_{\text {Train }}$ are due to the tail effects in linear convolution. We may decompose the discrete channel impulse response $h(t, \tau)$ into $N_{s}$ parallel symbol-rate sampled subchannel responses [18]. The $l^{\text {th }}$ received sample of the $\gamma^{\text {th }}$ sub-channel may be written as

$$
y_{l}^{(\gamma)}=\sum_{\mu=-L_{p} / 2+l}^{L_{c}+L_{p} / 2+l} d_{\mu} h_{l, l-\mu}^{(\gamma)}+n_{l}^{(\gamma)},
$$

where $y_{l}^{(\gamma)}=y\left(\left(l N_{s}+\gamma\right) T_{s}\right), n_{l}^{(\gamma)}=n\left(\left(l N_{s}+\gamma\right) T_{s}\right)$, and $h_{l, l-\mu}^{(\gamma)}=h\left(\left(l N_{s}+\gamma\right) T_{s},\left((l-\mu) N_{s}+\gamma\right) T_{s}\right)$, for $\gamma=$ $0,1, \ldots, N_{s}-1$. We may then write the $l^{t h}$ GLMS update equation for the $\gamma^{\text {th }}$ sub-channel as [14]

$$
\widehat{\mathbf{h}}_{l+1 \mid l}^{(\gamma)}=\mathbf{U}\left(\widehat{\mathbf{h}}_{l \mid l-1}^{(\gamma)}+\widehat{\mathbf{P}}^{(\gamma)} \mathbf{d}_{l}^{H}\left(y_{l}^{(\gamma)}-\widehat{y}_{l \mid l-1}^{(\gamma)}\right)\right),
$$

where the matrix $\mathbf{U}$ is defined as

$$
\mathbf{U}=\left(\begin{array}{cccc|c}
\mathcal{U}_{1} & \mathcal{U}_{2} & \ldots & \mathcal{U}_{P_{G}-1} & \mathcal{U}_{P_{G}} \\
\hline & \mathbf{I}_{L_{h}\left(P_{G}-1\right)} & \mathbf{0}_{L_{h}\left(P_{G}-1\right), L_{h}}
\end{array}\right),
$$

$\mathcal{U}_{r}=u_{r} \mathbf{I}_{L_{h}}$ and $u_{r}$ is the $r^{t h}$ tap weight of a polynomial predictor $^{3}$ of length $P_{G}$ and order $N_{G}<P_{G}$ [14], [15]. The matrix $\widehat{\mathbf{h}}_{l \mid l-1}^{(\gamma)}=\left[\widehat{\mathcal{H}}_{l \mid l-1}^{(\gamma) T}, \widehat{\mathcal{H}}_{l-1 \mid l-1}^{(\gamma) T}, \ldots, \widehat{\mathcal{H}}_{l-P_{G}+1 \mid l-1}^{T}\right]^{(\gamma) T}$ is the $l^{\text {th }}$ estimate of the channel state vector $\mathbf{h}_{l \mid l-1}^{(\gamma)}$ based on $(l-1)$ prior received samples, with $\mathcal{H}_{l \mid l-1}^{(\gamma)}=$ $\left[h_{l \mid l-1,-L_{p} / 2}^{(\gamma)}, \ldots, h_{l \mid l-1,0}^{(\gamma)}, \ldots, h_{l \mid l-1, L_{p} / 2+L_{c}-1}^{(\gamma)}\right]^{T} \cdot \widehat{\mathbf{P}}^{(\gamma)} \quad$ is the intermediate matrix and $\mathbf{d}_{l}=\left[d_{l+L_{c}+L_{p} / 2}, \ldots, d_{l+1}, d_{l}\right.$, $\left.d_{l-1}, \ldots, d_{l-L_{p} / 2} \mid \mathbf{0}_{1, L_{h}\left(P_{G}-1\right)}\right]$ is the data vector corresponding to the $l^{\text {th }}$ received sample. Note that $\widehat{\mathbf{P}}^{(\gamma)}$ can be obtained from an offline recursion based on the polynomial structure and is used in the estimation algorithm in place of the usual covariance matrix [14].

In data transmission mode, we modify the notation for the received samples as the channel is assumed constant over the $j^{\text {th }}$ transmission block and the training sequence is now embedded in the UW prefix. The $l^{t h}$ received sample of the $\gamma^{\text {th }}$ sub-channel for the $j^{\text {th }}$ block is written as

$$
y_{j, l}^{(\gamma)}=\sum_{\mu=-L_{p} / 2+l}^{L_{c}+L_{p} / 2+l} x_{j, \mu} h_{j, l-\mu}^{(\gamma)}+n_{j, l}^{(\gamma)},
$$

This assumes $N_{s}=2$, samples $y_{j,-N_{U W} / 2}^{(0)}, y_{j,-N_{U W} / 2}^{(1)}$ are used to provide the $\left(N_{\text {Train }}+j\right)^{t h}$ update of the channel. Since the UW sequence is known at the receiver for each transmission, the vector $\mathbf{d}_{j}$ for the $\left(N_{\text {Train }}+j\right)^{\text {th }}$ update is given by $\mathbf{d}_{j}=\left[x_{j,-\left(N_{U W}+L_{p}\right) / 2+L_{h}}, \ldots, x_{j,-N_{U W} / 2}, \ldots\right.$, $\left.x_{j,-\left(N_{U W}+L_{p}\right) / 2} \mid \mathbf{0}_{1, L_{h}\left(P_{G}-1\right)}\right]$.

\footnotetext{
${ }^{3}$ The polynomial weights $\left\{u_{r}\right\}$ are fixed and easily found [15].
}

\section{B. FD-TLE Algorithm}

We briefly describe the development of the equalizer filter matrix $\mathbf{D}_{T L E}[\psi]$ for the $\psi^{t h}$ iteration of the turbo equalization process. We initially assume the channel impulse response (CIR) is perfectly known at the receiver, and derive $\mathbf{D}_{T L E}[\psi]$ following [10]. We then extend this by deriving $\mathbf{D}_{T L E}[\psi]$ for the case of estimated CIR.

At the start of the $\psi^{\text {th }}$ iteration of the turbo equalization process, we denote the data APPs produced by the SISO decoder as $A P P_{n, l}[\psi-1]=P\left\{x_{n}=\mathcal{X}_{l} \mid \widehat{x}_{l}\right\}$. When $\psi=1$, the APP values are not available and are set to be equal. We define the zero-mean vectors $\widetilde{\mathbf{X}}_{N}[\psi]=\mathbf{X}_{N}-E_{\mathbf{x}_{N}, \psi-1}\left\{\mathbf{X}_{N}\right\}$, $\widetilde{\mathbf{X}}_{2 N}[\psi]=\mathbf{X}_{2 N}-E_{\mathbf{x}_{N}, \psi-1}\left\{\mathbf{X}_{2 N}\right\}$ and the modified received vector [10]

$$
\tilde{\mathbf{Y}}_{2 N}[\psi]=\mathbf{Y}-\mathbf{R} E_{\mathbf{x}_{N}, \psi-1}\left\{\mathbf{X}_{2 N}\right\}=\mathbf{R} \widetilde{\mathbf{X}}_{2 N}[\psi]+\mathbf{V},
$$

where $\mathbf{R}$ is defined following (5) and

$$
E_{\mathbf{x}_{N}, \psi-1}\left\{x_{n}\right\}=\sum_{l=0}^{M-1} A P P_{n, l}[\psi-1] \mathcal{X}_{l}
$$

is the statistical average of $x_{n}$ with respect to the data vector $\mathbf{x}_{N}$ evaluated on the basis of the APPs produced by the SISO decoder employing the BCJR algorithm [19], after $\psi-1$ iterations.

We next define the modified FD error vector

$$
\widetilde{\Delta}_{T L E}[\psi]=\mathbf{D}_{T L E}[\psi] \widetilde{\mathbf{Y}}[\psi]-\widetilde{\mathbf{X}}_{N}[\psi],
$$

To find $\mathbf{D}_{T L E}[\psi]$, we use the orthogonality principle [11], which in the FD is given by

$$
E\left\{\widetilde{\Delta}_{T L E}[\psi] \widetilde{\mathbf{Y}}[\psi]^{H}\right\}=\mathbf{0}_{N \times 2 N}
$$

1) Known CIR: Assuming perfect CSI is available and substituting (10) and (12) into (13), we obtain the equalizer matrix [10] as

$$
\mathbf{D}_{T L E}[\psi]=\left(\mathbf{I}_{N}-\boldsymbol{\Phi}[\psi]\right) \mathbf{J} \mathbf{R}^{H} \mathbf{K}_{T}[\psi],
$$

where $\mathbf{J}=\left[\mathbf{I}_{N} \mid \mathbf{I}_{N}\right]$,

$$
\begin{aligned}
\boldsymbol{\Phi}[\psi] & =E_{\mathbf{x}_{N}, \psi-1}\left\{\mathbf{X}_{N}\right\} E_{\mathbf{x}_{N}, \psi-1}\left\{\mathbf{X}_{N}^{H}\right\}, \\
\mathbf{K}_{T}[\psi] & =\left[\mathbf{R J}^{H}\left(\mathbf{I}_{N}-\boldsymbol{\Phi}[\psi]\right) \mathbf{J R}^{H}+\sigma_{n}^{2} \mathbf{I}_{2 N}\right]^{-1} .
\end{aligned}
$$

The data APPs are used to evaluate $\Phi[\psi]=\left[\phi_{i, p}[\psi]\right]$, where

$$
\begin{aligned}
\phi_{i, p}[\psi]= & \frac{1}{N} \sum_{n=0}^{N-1} E_{\mathbf{x}_{N}, \psi-1}\left\{\mathbf{x}_{n}\right\} \exp \left(-j \frac{2 \pi i n}{N}\right) \\
& \cdot \sum_{l=0}^{N-1} E_{\mathbf{x}_{N}, \psi-1}\left\{\mathbf{x}_{l}\right\}^{*} \exp \left(-j \frac{2 \pi p l}{N}\right) .
\end{aligned}
$$

2) Estimated CIR: We now consider the FD-TLE equations when only estimated CIR is available. We first replace the CIR $c(t)$ with the estimated CIR $\hat{c}(t)$. We then let $\mathbf{R}=\widehat{\mathbf{R}}-\mathbf{E}$, where $\mathbf{E}$ is the estimation error matrix. Using $\widehat{\mathbf{R}}-\mathbf{E}$ in place of $\mathbf{R}$ in (10), we obtain the equalizer matrix in terms of $\widehat{\mathbf{R}}$,

$$
\mathbf{D}_{T L E}[\psi]=\left(\mathbf{I}_{N}-\boldsymbol{\Phi}[\psi]\right) \mathbf{J} \widehat{\mathbf{R}}^{H} \mathbf{K}_{T}[\psi],
$$

where the filter matrix is given by

$$
\begin{aligned}
\mathbf{K}_{T}[\psi]= & {\left[\widehat{\mathbf{R}} \mathbf{J}^{H}\left(\mathbf{I}_{N}-\mathbf{\Phi}[\psi]\right) \mathbf{J} \widehat{\mathbf{R}}^{H}\right.} \\
& \left.+E\left\{\mathbf{E} \mathbf{E}^{H}\right\} \mathbf{J}^{H}\left(\mathbf{I}_{N}-\mathbf{\Phi}[\psi]\right) \mathbf{J}+\sigma_{n}^{2} \mathbf{I}_{2 N}\right]^{-1} .
\end{aligned}
$$


The Fourier transform of the estimated channel response can be written as

$$
\begin{aligned}
\hat{C}(f) & =\sum_{n=0}^{L_{c}-1} c_{n} \exp \left(-j 2 \pi f \tau_{n}\right)+\sum_{n=0}^{L_{c}-1} e_{n} \exp \left(-j 2 \pi f \tau_{n}\right) \\
& =C(f)+E(f),
\end{aligned}
$$

where $\tau_{n}, c_{n}$ and $e_{n}$ are the delay, gain and estimation error associated with the $n^{\text {th }}$ tap. Since $\hat{\boldsymbol{R}}=\boldsymbol{R}+\boldsymbol{E}$ each element of the diagonal matrix $\boldsymbol{E}$ can be obtained by sampling the estimation error component $E(f)$. Assuming that the estimation errors associated with each channel tap $\left\{e_{0}, \cdots, e_{L_{c}-1}\right\}$ are independent, we obtain

$$
\begin{gathered}
E\left\{\left(\sum_{n=0}^{L_{c}-1} e_{n} \exp \left(-j 2 \pi f \tau_{n}\right)\right)\left(\sum_{n=0}^{L_{c}-1} e_{n} \exp \left(-j 2 \pi f \tau_{n}\right)\right)^{*}\right\} \\
=\sum_{n=0}^{L_{c}-1} E\left\{e_{n} e_{n}^{*}\right\}
\end{gathered}
$$

This was derived assuming a rectangular pulse shape. A (root) raised cosine pulse response has $P(f) \leq 1$ meaning the components of the diagonal matrix $E\left\{\mathbf{E E}^{H}\right\}$ are upper bounded by $\sum_{n=0}^{L_{c}-1} E\left\{e_{n} e_{n}^{*}\right\}$. To evaluate $E\left\{\mathbf{E E}^{H}\right\}$ we require ideal CSI, which is not easily achieved in practice. However, simulation has found that $\sum_{n=0}^{L_{c}-1} E\left\{e_{n} e_{n}^{*}\right\}$ is small in magnitude, especially at high SNR [16].

The term $E\left\{\mathbf{E E}^{H}\right\} \mathbf{J}^{H}\left(\mathbf{I}_{N}-\boldsymbol{\Phi}[\psi]\right) \mathbf{J}$ in (19) will be small since elements in $E\left\{\mathbf{E E}{ }^{H}\right\}$ are small. Hence, we may set this term to zero and approximate (19) as

$$
\mathbf{K}_{T}[\psi] \cong\left[\widehat{\mathbf{R}} \mathbf{J}^{H}\left(\mathbf{I}_{N}-\mathbf{\Phi}[\psi]\right) \mathbf{J} \widehat{\mathbf{R}}^{H}+\sigma_{n}^{2} \mathbf{I}_{2 N}\right]^{-1} .
$$

We note that (22) has the same form as (16), which was obtained assuming ideal CSI. However, since $\widehat{\mathbf{R}}=\mathbf{R}+\mathbf{E}$ is used in (22) instead of the actual composite channel $\mathbf{R}$, degradation in receiver performance is expected due to the estimation error embedded in $\widehat{\mathbf{R}}$. This in effect causes the equalizer to be mismatched to the channel response.

\section{Simulation Results}

We consider two channel models, one typical of mobile and one of fixed wireless systems. The first is an 11-TAP mobile wireless model that follows from [20], [21]. It assumes no line-of-sight path and uses 11 equally spaced taps with $1 \mu s$ spacing, the assumed symbol period. The delay power profile for this channel, $Q_{\text {Mobile }}(\tau)$, is set to be a truncated exponential function with mean $2.5 \mu \mathrm{s}$ and maximum delay $\tau_{\max }=10 \mu \mathrm{s}$. The SUI-5 model [22] has 3 taps with relative tap powers of $0 \mathrm{~dB},-5 \mathrm{~dB}$, and $-10 \mathrm{~dB}$, respectively, and a total delay spread of $10 \mu \mathrm{s}$. In this case the maximum Doppler frequency is $2.5 \mathrm{~Hz}$, which represents very slow fading. However, the SUI-5 model is very dispersive. In both cases, we consider the channel to be quasi-static within a data frame and only vary from frame to frame.

All simulations assume the symbol period is $T=1 \mu \mathrm{s}$ and the root-raised cosine pulse shape $p(t)$ has a rolloff of $\alpha=0.4$ truncated to $L_{p}=10$ symbol intervals. The DFT order is $2 N=2048$. A 64 -by-16 $(N=1024)$ block interleaver is

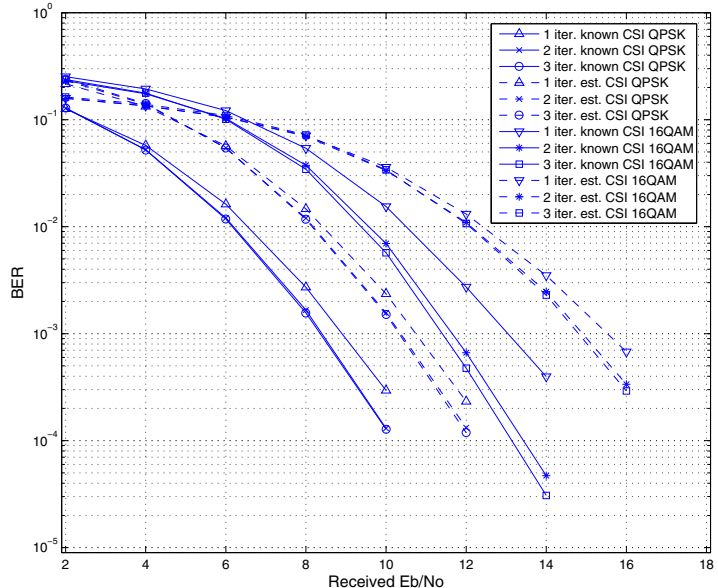

(a) For 1/2-rate 4-state $(5,7)$ convolutional code.

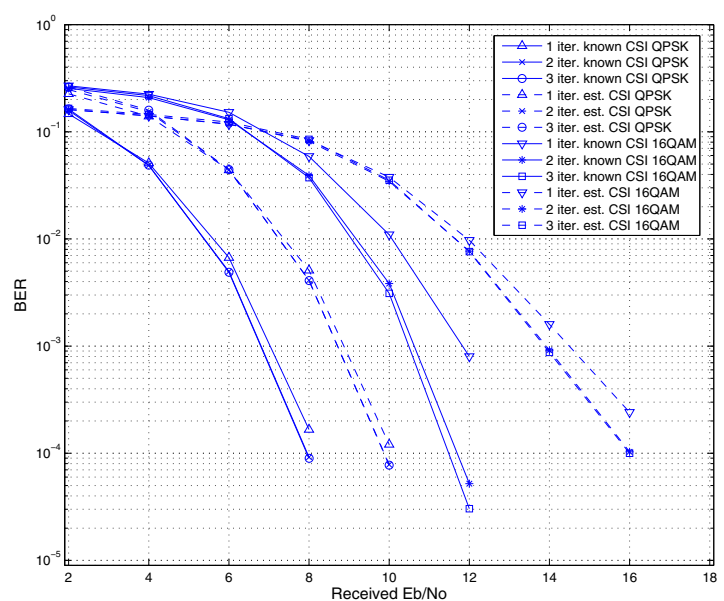

(b) For 1/2-rate 64-state $(133,171)$ convolutional code.

Fig. 2. Overall system performance for the 11-TAP channel.

used. The GLMS channel estimator length and polynomial order are set to $P_{G}=3$ and $N_{G}=1$, respectively. We found from simulations that this provided the best performance.

The signal-to-noise ratio (SNR) is defined as $10 \log \left(E_{b} / N_{0}\right)$, where $E_{b}=\frac{E_{s}}{\mathcal{R} \cdot \log _{2} M}$ is the average received energy per data bit, $E_{s}$ is the average received symbol energy and $\mathcal{R}$ is the rate of the convolutional code ${ }^{4}$.

The bit-error-rate (BER) for the 11-TAP channel using the 4-state $(5,7)$ and 64 -state $(133,171)$ convolutional codes is shown in Fig. 2. As in [10], most of the gain is obtained using two iterations. The gain is smaller in the case of estimated CSI. For the 4-state code, there is a degradation due to imperfect CSI of approximately $2 \mathrm{~dB}$ for QPSK and $4 \mathrm{~dB}$ for 16-QAM at $10^{-4}$ BER after 3 iterations. Similar degradation is observed for the 64-state code. This is expected since imperfect CSI introduces errors into the equalization process.

The BER for the SUI-5 channel with the 4-state $(5,7)$ and

${ }^{4} \mathcal{R}$ is the convolutional code rate for the data block. The UW sequences are appended to the data block after encoding. So, $\mathcal{R}$ does not include the throughput loss introduced by the UW sequences [16]. 


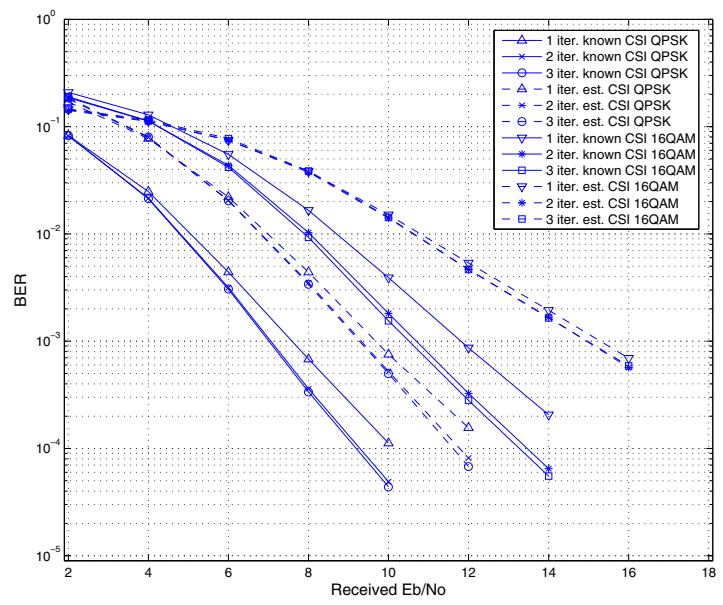

(a) For 1/2-rate 4-state $(5,7)$ convolutional code.

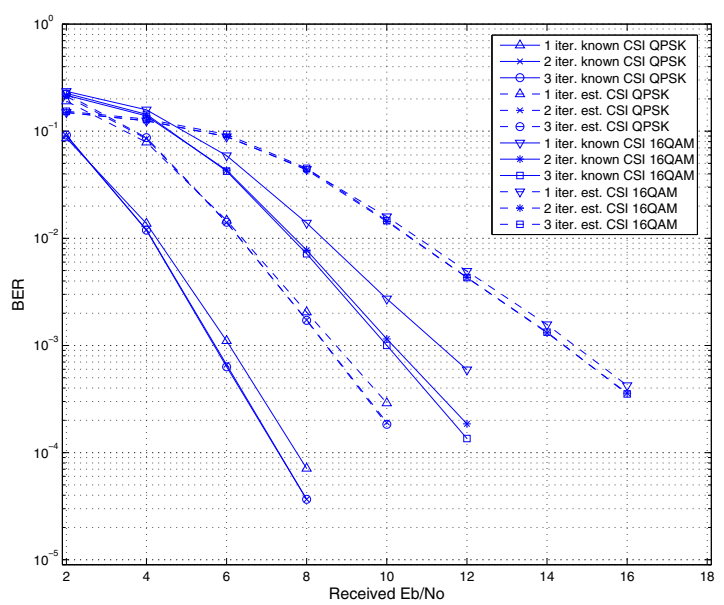

(b) For 1/2-rate 64-state $(133,171)$ convolutional code.

Fig. 3. Overall system performance for the SUI-5 channel.

64-state $(133,171)$ convolutional code is shown in Fig. 3. The results illustrate that when using estimated CSI, the FD-TLE receiver improves performance using a second iteration, but nothing further is gained from a third iteration. When the 4state $(5,7)$ convolutional code is used, there is a performance degradation due to imperfect CSI of approximately $2 \mathrm{~dB}$ at a BER of $10^{-4}$ for QPSK and $4 \mathrm{~dB}$ at a BER of $5 \times 10^{-4}$ for $16-$ QAM, after 3 iterations. When the 64-state code is used, there is a performance degradation of approximately $3 \mathrm{~dB}$ at a BER of $10^{-4}$ for QPSK and $5 \mathrm{~dB}$ at a BER of $5 \times 10^{-4}$ for 16-QAM after 3 iterations. As with the 11-TAP channel, the gain is less than for perfect CSI. Note that performance improvement due to iteration is smaller for estimated CSI. This suggests that the channel estimation error is significant to the turbo equalization process. Hence, accurate CSI is required to obtain significant improvement from the iterative process.

\section{CONClusion}

A FD turbo linear equalizer has been described, which estimates CSI in the TD. It utilizes the FD-TLE scheme of [10] and the channel estimator of [14]. A derivation of the modified FD-TLE algorithm employing estimated CSI was also presented. BER performance of the system has been evaluated. The results illustrate that most of the achievable gain is obtained after 2 iterations. The results show that the system may be applied to different wireless channels. Lastly, the results clearly show the necessity of using accurate CSI in the turbo equalization process. This suggests that a more accurate channel estimator, such as the GRLS structure [15] or a Kalman filter [17] should be used, which would lead to increased system complexity.

\section{REFERENCES}

[1] D. Falconer, S. Ariyavisitakul, A. Benjamin-Seeyar, and A. Edison, "Frequency domain equalization for single-carrier broadband wireless systems," IEEE Commun. Magazine, vol. 40, pp. 58-66, Apr. 2002.

[2] C. Douillard, A. Picart, M. Jezequel, P. Didier, C. Berrou, and A. Galvieux, "Iterative correction of intersymbol interference: Turboequalization," Euro. Trans. Commun., vol. 6, pp. 507-511, Sep. 1995.

[3] M. Tüchler and J. Hagenauer, "Turbo equalization using frequency domain equalizers," in Proc. Allerton Conf., vol. 4, pp. 1234-1243, Oct. 2000.

[4] M. Tüchler and J. Hagenauer, "Linear time and frequency domain turbo equalization," in Proc. VTC, vol. 4, pp. 2773-2777, Oct. 2001.

[5] M. Tüchler, A. Singer, and R. Koetter, "Minimum mean squared error equalization using a priori information," IEEE Trans. Signal Processing, vol. 50, pp. 673-683, Mar. 2002.

[6] M. Tüchler, R. Koetter, and A. Singer, "Turbo equalization: Principles and new results," IEEE Trans. Commun., vol. 50, pp. 754-767, May. 2002.

[7] M. Tüchler, R. Koetter, and A. Singer, "Turbo equalization," IEEE Signal Processing Magazine, vol. 20, pp. 67-80, Oct. 2004.

[8] R. Otnes and M. Tüchler, "On iterative equalization, estimation, and decoding," in Proc. ICC, vol. 4, pp. 1918-1923, May. 2004.

[9] R. Otnes and M. Tüchler, "Iterative channel estimation for turbo equalization of time-varying frequency-selective channels," IEEE Trans. Commun., vol. 3, pp. 2958-2962, Nov. 2003.

[10] F. Pancaldi and G. Vitetta, "Block channel equalization in the frequency domain," IEEE Trans. Commun., vol. 53, pp. 463-471, Mar. 2005.

[11] S. Haykin, Adaptive Filter Theory, 3rd Edition. Prentice-Hall: AddisonWesley, 1996.

[12] J. J. Shynk, "Frequency-domain and multirate adaptive filtering," IEEE Signal Processing Magazine, vol. 9, pp. 15-37, Jan. 1992.

[13] S. Song, A. Singer, and K.-M. Sung, "Soft input channel estimation for turbo equalization," IEEE Trans. Signal Processing, vol. 52, pp. III2805-III-2808, Oct. 2004.

[14] W. Leon and D. Taylor, "The polynomial-based generalized least mean squares estimator for rician and rayleigh fading channels," in Proc. VTCFall, vol. 6, pp. 3861-3865, 2004.

[15] W. Leon and D. Taylor, "The polynomial-based generalized recursive least squares estimator for rayleigh fading channels," in Proc. GLOBECOM, vol. 5, pp. 2401-2405, 2003.

[16] F. Shiao, Combined Channel Estimation and Turbo Equalization on Wireless Channels. University of Canterbury, Christchurch, New Zealand: M.E. Thesis, 2007.

[17] Q. Dai and E. Shwedyk, "Detection of bandlimited signals over frequency selective rayleigh fading channels," IEEE Trans. Commun., vol. 42, pp. 941-950, Mar. 1994.

[18] D. Borah and B. Hart, "Frequency-selective fading channel estimation with a polynomial time-varying channel model," IEEE Trans. Commun., vol. 47, pp. 862-873, Jun. 1999.

[19] L. Bahl, J. Cocke, F. Jelinek, and J. Riviv, "Optimal decoding of linear codes for minimizing symbol error rate," IEEE Trans. Inform. Theory, vol. 20, pp. 284-287, Mar. 1974.

[20] W. Jakes, Microwave Mobile Communications. New York: IEEE Press, 1974.

[21] M. Luise, R. Reggiannini, and G. Vitetta, "Blind equalization/detection for OFDM signals over frequency-selective channels," IEEE Commun. Magazine, vol. 40, pp. 58-66, Apr. 2002.

[22] V. Erceg and et al, "Channel models for fixed wireless applications," IEEE 802.16a cont. IEEE 806.16.3c-01/29r1, Feb. 2001. 